Pacific Journal of Mathematics

ORTHOGONAL PROJECTIONS ONTO SUBSPACES OF THE 


\title{
ORTHOGONAL PROJECTIONS ONTO SUBSPACES OF THE HARMONIC BERGMAN SPACE
}

\author{
Emil J. Straube
}

\begin{abstract}
Let $\Omega \subset \mathbf{R}^{m}$ be a bounded, smooth domain. We construct a continuous linear operator $T: W^{0}(\Omega) \rightarrow W^{0}(\Omega)$ which for all $k \in(\mathbf{N} \cup\{\infty\})$ is actually continuous from $W^{k}(\Omega) \rightarrow W_{0}^{k}(\Omega)$, and which moreover has the property that $S T=S$, for any orthogonal projection $S$ of $W^{0}(\Omega)$ onto a subspace of the harmonic Bergman space. That is, the operator assigns to each function a function vanishing to high (infinite if $k=\infty$ ) order at $b \Omega$, but with the same projection. $S$ can in particular be the harmonic Bergman projection, or, when $\Omega \subset \mathrm{C}^{n}$, the (analytic) Bergman projection. The question whether such an operator exists arises for example in connection with regularity properties of the Bergman projection and their intimate connection with boundary regularity of holomorphic mappings.
\end{abstract}

1. Introduction and results. Let $\Omega \subset \mathbf{R}^{m}$ be a bounded domain with smooth boundary. For $k \in \mathbf{N}$, we denote by $W^{k}(\Omega)$ the usual Sobolev spaces of order $k$ on $\Omega$ (see [10]), and by $W_{0}^{k}(\Omega)$ the closure of $C_{0}^{\infty}(\Omega)$ in $W^{k}(\Omega) . h^{k}(\Omega)$ denotes the closed subspace of $W^{k}(\Omega)$ consisting of harmonic functions; the harmonic Bergman projection $Q$ is the orthogonal projection of $W^{0}(\Omega)\left(=\mathscr{L}^{2}(\Omega)\right)$ onto $h^{0}(\Omega)$. We are interested in projections onto subspaces of $h^{0}(\Omega)$. The most interesting examples will be $Q$ itself and, in the case where $\Omega$ lies in complex euclidean space $\mathbf{C}^{n} \cong \mathbf{R}^{2 n}$, the Bergman projection $P$. This is the orthogonal projection of $W^{0}(\Omega)$ onto $A^{0}(\Omega)$, the subspace of $W^{0}(\Omega)$ consisting of analytic functions. The purpose of the present paper is to construct a continuous linear operator $T$ from $W^{0}(\Omega)$ to $W^{0}(\Omega)$, which to each function in $W^{k}(\Omega)$ assigns a function in $W_{0}^{k}(\Omega)$ (i.e. "vanishing to order $k-1$ "), but with the same projection. More precisely, we have

THEOREM 1.1. Let $\Omega$ as above. There is a continuous linear operator T: $W^{0}(\Omega) \rightarrow W^{0}(\Omega)$ which satisfies

(i) for all $k \in(\mathbf{N} \cup\{\infty\})$, T maps $W^{k}(\Omega)$ continuously into $W_{0}^{k}(\Omega)$.

(ii) if $S$ is the orthogonal projection of $W^{0}(\Omega)$ onto an arbitrary closed subspace of $h^{0}(\Omega)$, then

$$
S T=S
$$


REMARK 1.2. The point of the theorem is really that there exists $T$ with (i) and such that $Q T=Q$. For $S$ as in the theorem, $S=S Q$, so that then trivially $S T=S Q T=S Q=S$.

REMARK 1.3. By the standard interpolation argument, $T$ is continuous from $W^{r}(\Omega)$ to $W_{0}^{r}(\Omega)$, for $r$ real, $\geq 0$. For $r=$ integer $+1 / 2$, we even get continuity from $W^{r}(\Omega)$ to $W_{00}^{r}(\Omega)$; see [10] for details and definition of the last space.

REMARK 1.4. The smoothness of $T g$ depends only on the smoothness of $g$ near $b \Omega$. More precisely: if $g \in W^{k}(\Omega \backslash K)$ for some compact subset $K$, then $T g \in W_{0}^{k}(\Omega)$. This will be clear from the proof of Theorem 1.1.

The main source of motivation for constructing operators like $T$ are questions revolving about the Bergman projection $P$, and its intimate connection with boundary behavior of holomorphic mappings ([4] and its references, [7]). One of the key steps was Bell's construction of (differential) operators $\varphi^{k}: W^{k+N(k)}(\Omega) \rightarrow W_{0}^{k}(\Omega)$, such that $P \varphi^{k}=P . \varphi^{k}$ is also bounded from $A^{k}(\Omega) \rightarrow W_{0}^{k}(\Omega)\left(A^{k}(\Omega)\right.$ is the analytic subspace of $W^{k}(\Omega)$ ). A revised version of these operators is in [1]. Harmonic and pluriharmonic versions were given in [2] and [3], respectively. From this circle of ideas, the question also arises whether the conditions $R^{k}: P$ maps $W^{k}(\Omega)$ into itslef, and $R_{0}^{k}: P$ maps $W_{0}^{k}(\Omega)$ into $W^{k}(\Omega)$, are equivalent. The $\varphi^{k}$ do not give an answer. This question also arises from [9], where $R_{0}^{k}$ rather than $R^{k}$ appeared naturally. The question was answered affirmatively by the author in [12]. It was shown that there exist continuous operators $T^{k}: W^{k}(\Omega) \rightarrow W_{0}^{k}(\Omega)$, such that $P T^{k}=P$. For $k=\infty$, it was shown in [6] and [12] that for $g \in W^{\infty}(\Omega)$, there is always $h \in W_{0}^{\infty}(\Omega)$ with $P h=P g$, but it was not clear whether the function in $W_{0}^{\infty}(\Omega)$ could be chosen in a continuous, linear way (in [12] a continuous linear map into a quotient of $W_{0}^{\infty}(\Omega)$ was obtained). The operator $T$ gives a unified approach to all the above. In addition, we write down $T$ quite explicitely (in contrast to [12], where the author's $T^{k}$ were obtained by abstract arguments). This clarifies the situation; in fact it is precisely this feature which allows to check the regularity properties. Note that the equivalence for the harmonic Bergman projection, corresponding to $R^{k} \Leftrightarrow R_{0}^{k}$, gives nothing interesting, because $Q$ always maps $W^{k}(\Omega)$ into itself ([2]). In intermediate cases however, such as the projection onto the pluriharmonic functions, the corresponding equivalence (also obtained from $T$ ) is of interest. 
We also briefly mention that the operator $T$ may be used to obtain equivalence of certain negative Sobolev norms on harmonic functions (no geometric assumptions on $\Omega$ ), compare [5], §4. We do not elaborate on this, because this equivalence also follows directly from the Sobolev estimate on an "improper" $\mathscr{L}^{2}$-pairing given in [12], and it is our opinion that the approach via the pairing is more natural in that context.

The construction of the operator $T$ rests upon an observation about the projections, which we now proceed to state. It says, loosely speaking, that the condition of having the same projection as some given function may be reformulated as a certain boundary condition. Consider the Dirichlet problem for the Laplacian

$$
\begin{aligned}
\Delta h=g & \text { on } \Omega \\
h=0 & \text { on } b \Omega,
\end{aligned}
$$

with $g \in W^{k}(\Omega)$, so that the solution $h \in W^{k+2}(\Omega)$. Let $\Psi$ be any function in $W^{k+2}(\Omega)$ such that

$$
\begin{aligned}
\Psi & =0 \\
\frac{\partial \Psi}{\partial \nu} & =\frac{\partial h}{\partial \nu} \quad \text { on } b \Omega
\end{aligned}
$$

Here, $\partial / \partial \nu$ denotes the normal derivative (normal to $b \Omega$, oriented inward); the boundary values are, as usual, to be understood as traces. Then we have

Proposition 1.5. Let $g \in W^{k}(\Omega)$ and let $h$ be the solution of the Dirichlet problem (2). For $\Psi$ satisfying (3),

$$
S(\Delta \Psi)=S g
$$

for any projection $S$ as in Theorem 1.1 .

As we shall see, the main point here is that (4) is implied by a boundary condition on $\Psi$ (namely (3)).

Proposition 1.5 allows essentially to reduce the problem to finding functions with prescribed normal derivatives on $b \Omega$. However, infinitely many derivatives will be involved, and in order to get extensions depending linearly on the data, special care has to be taken. In $\S 3$, we construct sequences of extension operators (roughly one operator for each normal derivative) whose norms are well controlled. For certain boundary data spaces (including the ones arising from our problem), they can be summed up to yield a linear operator which gives functions with the infinitely many prescribed normal derivatives. The construction is an infinite version of a construction in [8]; it is also somewhat motivated by the construction in [11]. 
2. Proofs. We first prove Proposition 1.5. We have

$$
\Delta \Psi-g=\Delta(\Psi-h) \text {. }
$$

Use (2) and (3) (\$1) to conclude that $\Psi-h=\partial(\Psi-h) / \partial \nu=0$ on $b \Omega$ so that $\Psi-h \in W_{0}^{2}(\Omega)$ ([10]). Therefore, $\Delta(\Psi-h)$ is orthogonal to $h^{0}(\Omega)$ (integrate by parts, no boundary terms appear). Thus, by (1), $\Delta \Psi-g$ is orthogonal to $h^{0}(\Omega)$ and thus to the image of $S$, whence the result.

Now we prove Theorem 1.1. Let $L: W^{0}(\Omega) \rightarrow W^{2}(\Omega)$ be the solution operator of the Dirichlet problem

$$
\begin{aligned}
\Delta L g=g & \text { on } \Omega \\
L g=0 & \text { on } b \Omega .
\end{aligned}
$$

For $g \in W^{0}(\Omega), T g$ will be defined as $\Delta \Psi$, for suitable $\Psi$, with

$$
\begin{aligned}
\Psi & =0 \\
\frac{\partial \Psi}{\partial \nu} & =\frac{\partial}{\partial \nu} L g \quad \text { on } b \Omega
\end{aligned}
$$

(3) will ensure, by Proposition 1.5, that $S \Delta \Psi=S g$. The condition that $\Delta \Psi=T g \in W_{0}^{k}(\Omega)$, may also be formulated as a boundary condition; it is equivalent to the condition

$$
\frac{\partial^{J}}{\partial \nu^{j}} \Delta \Psi=0 \quad \text { on } b \Omega, \quad 0 \leq j \leq k-1,
$$

see [10]. The next step is to observe that (3) and (4) together may equivalently be written by prescribing only normal derivatives of $\Psi$. It will be convenient to work in local coordinates near $b \Omega$, so we choose a partition of unity $\left\{\varphi_{s}\right\}$ of $b \Omega$, so that supp $\varphi_{s}$ is contained in a coordinate neighborhood $U_{s}$ of $b \Omega$, which is "small" so that its local coordinates $\left(\theta_{1}, \ldots, \theta_{m-1}\right)$ together with $\nu$ (signed distance to $b \Omega$ ) can be used as coordinates of $\mathbf{R}^{m}$ near $U_{s}$. Then the task is reduced (by linearity) to constructing $\Psi_{s} \in W^{k+2}(\Omega)$ satisfying the boundary condition

$$
\begin{aligned}
\Psi_{s} & =0 \\
\frac{\partial \Psi_{s}}{\partial \nu} & =\varphi_{s} \frac{\partial}{\partial \nu} L g \\
\frac{\partial^{J}}{\partial \nu^{J}} \Delta \Psi_{s} & =0 \quad 0 \leq j \leq k-1,
\end{aligned}
$$

whenever the function $g$ we start out with is in $W^{k}(\Omega)$. Near $U_{s}$, the Laplacian is expressed in the local coordinates (the index $s$ is suppressed) 
as

$$
\Delta=\frac{\partial^{2}}{\partial \nu^{2}}+\frac{1}{2 g} \frac{\partial g}{\partial \nu} \frac{\partial}{\partial \nu}+\frac{1}{\sqrt{g}} \sum_{i, j=1}^{m-1} \frac{\partial}{\partial \theta_{j}}\left(g^{i j} \sqrt{g} \frac{\partial}{\partial \theta_{l}}\right)
$$

where

$$
\begin{aligned}
g_{i j} & =\sum_{l=1}^{m} \frac{\partial x_{l}}{\partial \theta_{i}} \frac{\partial x_{l}}{\partial \theta_{j}}, \\
\left(g^{i j}\right) & =\left(g_{i j}\right)^{-1} \text { and } g=\operatorname{det}\left(g_{l j}\right) .
\end{aligned}
$$

Thus

$$
\frac{\partial^{J}}{\partial \nu^{j}} \Delta=\frac{\partial^{2+j}}{\partial \nu^{2+j}}+\frac{\partial^{j}}{\partial \nu^{j}}\left(\frac{1}{2 g} \frac{\partial g}{\partial \nu} \frac{\partial}{\partial \nu}+\sum_{i, l=1}^{m-1} \frac{\partial}{\partial \theta_{l}}\left(g^{l l} \sqrt{g} \frac{\partial}{\partial \theta_{i}}\right)\right) .
$$

Note that the second term on the right side of (7) involves only normal derivatives of order at most $j+1$. So if one knows the traces of $\Psi$ and $\partial \Psi / \partial \nu$ on $b \Omega$, and those of $\partial^{j} \Delta \Psi / \partial \nu^{j}$, one may recursively calculate the traces of $\partial^{j} \Psi / \partial \nu^{j}$. Thus we obtain a sequence $\left(B_{j}^{s}\right)_{j=2}^{\infty}$ of differential operators, such that for all $k \in \mathbf{N}$, the boundary condition

$$
\begin{aligned}
\Psi_{s} & =0 \\
\frac{\partial \Psi_{s}}{\partial \nu} & =\varphi_{s} \frac{\partial}{\partial \nu} L g \\
\frac{\partial^{j} \Psi_{s}}{\partial \nu^{j}} & =B_{J}^{s}\left(\varphi_{s} \frac{\partial}{\partial \nu} L g\right), \quad 2 \leq j \leq k+1
\end{aligned}
$$

is equivalent to the boundary condition (5) (in $U_{s}$ ). For convenience, we set $B_{0}^{s}:=0$ and $B_{1}^{s}=$ id (the identity operator), so that (8) can be rewritten as

$$
\frac{\partial^{j} \Psi_{s}}{\partial \nu^{j}}=B_{j}^{s}\left(\varphi_{s} \frac{\partial}{\partial \nu} L g\right), \quad 0 \leq j \leq k+1
$$

The order of $B_{j}^{s}$ does not exceed $j-1$, for $j \geq 1$. Since $B_{j}^{s}\left(\varphi_{s}(\partial L g / \partial \nu)\right)$ is compactly supported in $U_{s}$, we may continue it to $b \Omega$ by setting it equal to 0 outside $U_{s}$. In this way, (9) may be viewed as a boundary condition on all of $b \Omega$. Then, any $\Psi_{s}$ is $W^{k+2}(\Omega)$ which satisfies this condition, will also satisfy (5) on all of $b \Omega$ (in $U_{s}$ by construction of the $B_{j}^{s}$, on $b \Omega \backslash \operatorname{supp} \varphi_{s}$ because there all normal derivatives are 0 on $b \Omega$ ). We point out once more that the $B_{J}^{s}$ are easily calculated explicitly, by recursion, starting with $B_{2}^{s}$. 
In $\S 3$, we will for every given sequence $\left(\alpha_{j}\right)_{0}^{\infty}$ of positive numbers construct a sequence $\left(R^{j}\right)_{0}^{\infty}$ of linear operators defined on $C^{\infty}(b \Omega)^{\prime} \cong$ $\bigcup_{k=0}^{\infty} W^{-k}(b \Omega)$, such that for all $k \in \mathbf{N}$ :

(i)

$R^{j}$ is continuous from $W^{k-j-1 / 2}(b \Omega)$ to $W^{k}(\Omega) ;$

we denote the corresponding operator norm by $\left\|R^{j}\right\|_{k}$.

(ii) for $0 \leq s \leq k-1$,

$$
\left.\left(\frac{\partial^{s}}{\partial \nu^{s}} R^{j} u\right)\right|_{b \Omega}= \begin{cases}0 & \text { if } s \neq j \\ u & \text { if } s=j\end{cases}
$$

Note that these traces are well defined, since $R^{j} u \in W^{k}(\Omega)$.

$$
\sum_{j \geq 0}\left\|R^{j}\right\|_{k} \alpha_{j}<\infty
$$

This property is independent of the boundary norm used (see $\S 3$ ).

This will put us in a position to define the operator $T$. Denote by $C_{J k}^{s}$ the operator norm of

$$
B_{j}^{s}\left(\varphi_{s} \frac{\partial}{\partial \nu} L\right): W^{k}(\Omega) \rightarrow W^{k+3 / 2-j}(b \Omega) .
$$

We choose the sequence $\left(\alpha_{j}\right)_{0}^{\infty}$ with $\alpha_{j}:=\sup _{s} \sup _{k \leq j} C_{j k}^{s}$ and construct the $R^{j}$ corresponding to this sequence. Now we set for $g \in W^{0}(\Omega)$

$$
T g:=\Delta\left(\sum_{s} \sum_{j \geq 0} R^{j} B_{j}^{s}\left(\varphi_{s} \frac{\partial}{\partial \nu} L g\right)\right)
$$

$T$ is well defined: for $k \in \mathbf{N}$ fixed, we have

$$
\left\|R^{j} B_{j}^{s}\left(\varphi_{s} \frac{\partial}{\partial \nu} L g\right)\right\|_{W^{k+2}(\Omega)} \leq\left\|R^{J}\right\|_{k+2} C_{J k}^{s}\|g\|_{W^{k}(\Omega)}
$$

Therefore,

$$
\begin{aligned}
& \left\|\sum_{j \geq 0} R^{J} B_{J}^{s}\left(\varphi_{s} \frac{\partial}{\partial \nu} L g\right)\right\|_{W^{k+2}(\Omega)} \leq\left(\sum_{j \geq 0}\left\|R^{J}\right\|_{k+2} C_{j k}^{s}\right)\|g\|_{W^{k}(\Omega)} \\
& \quad \leq\left(\sum_{j=0}^{k}\left\|R^{j}\right\|_{k+2} C_{j k}^{s}+\sum_{j>k}\left\|R^{j}\right\|_{k+2} \alpha_{j}\right)\|g\|_{W^{k}(\Omega)} \\
& \quad \leq C(k)\|g\|_{W^{k}(\Omega)} .
\end{aligned}
$$


Here, we have used (12); also note that $s$ ranges only over finitely many integers. From (14) it follows that $T$ is continuous from $W^{k}(\Omega)$ to itself. If

$$
\Psi_{s}=\sum_{j \geq 0} R^{j} B_{j}^{s}\left(\varphi_{s} \frac{\partial}{\partial \nu} L g\right)
$$

and

$$
\Psi:=\sum_{s} \Psi_{s}
$$

we have to check (3) and (4), or, after what we've done, just (9). Let $k$ still fixed. Let $0 \leq t \leq k+1$. Then we calculate the traces

$$
\begin{aligned}
\frac{\partial^{t} \Psi_{s}}{\partial \nu^{t}} & =\sum_{j \geq 0} \frac{\partial^{t}}{\partial \nu^{t}} R^{j} B_{j}^{s}\left(\varphi_{s} \frac{\partial}{\partial \nu} L g\right) \\
& =\sum_{j \geq 0} \delta_{t j} B_{j}^{s}\left(\varphi_{s} \frac{\partial}{\partial \nu} L g\right)=B_{t}^{s}\left(\varphi_{s} \frac{\partial}{\partial \nu} L g\right) .
\end{aligned}
$$

This is (9). Thus $T$ has all the properties required in Theorem 1.1, except possibly those relating to the case $k=\infty$. However, these are a consequence of the properties for all $k$. Therefore, the proof of Theorem 1.1 is complete.

REMARK 2.1. There is an abstract argument which gives an operator $T^{\infty}: W^{\infty}(\Omega) \rightarrow W_{0}^{\infty}(\Omega)$, such that $S T^{\infty}=S$, for $S$ as in Theorem 1.1. We briefly indicate it, as it has some interest of its own. Again, it suffices to treat the case $S=Q$. Moreover, we only need to find a right inverse $\tilde{T}$ for $Q: W_{0}^{\infty}(\Omega) \rightarrow h^{\infty}(\Omega)$, then $T^{\infty}:=\tilde{T} Q$ will do the job, since $Q$ is continuous from $W^{\infty}(\Omega) \rightarrow h^{\infty}(\Omega)$ ([2]). To find this right inverse, consider the sequence

$$
0 \rightarrow \operatorname{ker} Q \rightarrow W_{0}^{\infty}(\Omega) \stackrel{Q}{\rightarrow} h^{\infty}(\Omega) \rightarrow 0 .
$$

It is exact (for the surjectivity of $Q$, see [12]). Now $h^{\infty}(\Omega) \cong C^{\infty}(b \Omega)$, by the Poisson extension, which in turn is isomorphic to $s$, the space of rapidly decreasing sequences ([14], Theorem 2.3). It is easy to show that $\operatorname{ker} Q \cong W_{0}^{\infty}(\Omega)$, which is again isomorphic to $s$ ([14], Theorem 2.3). Therefore, Vogt's splitting theorem ([13], Theorem 2.2, see also Theorem 1.3) applies and yields a continuous right inverse $\tilde{T}$ of $Q$. The drawback of this approach is that it does not yeild exact preservation of differentiability (measured in Sobolev norms) for $\tilde{T}$. 
3. Prescribing infinitely many normal derivatives. In this section, we construct the operators $R^{j}$ with properties (10), (11) and (12) of $\S 2$. With the help of a partition of unity and local flattenings of the boundary, we reduce the problem to the case where $\Omega$ is a euclidean half space, $\Omega=\left\{(x, t) \in \mathbf{R}^{m} \times \mathbf{R} / t>0\right\}$ ( $m$ here does not denote the same integer as in the previous sections). This causes no problems as far as properties (i) and (ii) (i.e. (10) and (11)) are concerned, but property (iii) needs some attention, since cutoffs affect norms. Also, $\left\|R^{j}\right\|_{k}$ depends on the boundary norms used, so it is not a priori clear that property (iii) is independent of the choice of norm for the boundary Sobolev spaces. However, for both problems the relevant norms are estimated by factors $C_{j k}$, and considering a new sequence $\tilde{\alpha}_{j}:=\left(\sup _{k \leq j} C_{j k}\right) \alpha_{j}$ (a similar “diagonal process' was used in \$2) shows that (iii) is preserved. With these considerations done, we only state the result in the setting of a euclidean half space.

THEOREM 3.1. Let $\left(\alpha_{j}\right)_{0}^{\infty}$ be an arbitrary sequence of positive numbers. Then there exists a sequence $\left(R^{j}\right)_{0}^{\infty}$ of operators defined on $W^{-\infty}\left(\mathbf{R}^{m}\right)$, valued in $W^{-\infty}(\Omega)$, such that for all $k \in \mathbf{N}$ :

(i)

(1) $R^{j}$ is continuous from $W^{k-j-1 / 2}\left(\mathbf{R}^{m}\right)$ to $W^{k}(\Omega), \quad j=0,1,2, \ldots$,

(ii) for $0 \leq s \leq k-1$,

$$
\left.\frac{\partial^{s}}{\partial t^{s}} R^{j} a\right|_{\mathbf{R}^{m}}=\left\{\begin{array}{ll}
0 & \text { if } s \neq j \\
a & \text { if } s=j
\end{array} \quad j=0,1,2, \ldots\right.
$$

(iii) If $\left\|\mathbf{R}^{j}\right\|_{k}$ denotes the operator norm of $R^{j}$ as an operator from $W^{k-j-1 / 2}\left(\mathbf{R}^{m}\right)$ to $W^{k}(\Omega)$, then

$$
\sum_{j \geq 0}\left\|R^{j}\right\|_{k} \alpha_{j}<\infty
$$

REMARK 3.2. We identify $b \Omega$ with $\mathbf{R}^{m}$. The left side of (2) is understood in the sense of traces. These are well defined for $0 \leq s \leq k-1$, since $R^{j} a \in W^{k}(\Omega)$.

Proof. First note that

(4) $W^{k}(\Omega)=\left\{u \mid u \in \mathscr{L}^{2}\left((0, \infty), W^{k}\left(\mathbf{R}^{m}\right)\right)\right.$,

$$
\left.\frac{\partial^{k}}{\partial t^{k}} u \in \mathscr{L}^{2}\left((0, \infty), W^{0}\left(\mathbf{R}^{m}\right)\right)\right\} .
$$


Here, $\mathscr{L}^{2}((0, \infty), E)$ denotes the space of square integrable $E$-valued functions on $(0, \infty)$, with norm

$$
\|u\|_{\mathscr{L}^{2}((0, \infty), E)}=\left(\int_{0}^{\infty}\|u(t)\|_{E}^{2} d t\right)^{1 / 2} .
$$

Equality in (4) means "same elements and equivalent norms", i.e.

$$
\|u\|_{W^{k}(\Omega)} \approx\left(\int_{0}^{\infty}\left(\|u(t)\|_{W^{k}\left(\mathbf{R}^{m}\right)}^{2}+\left\|\frac{\partial^{k}}{\partial t^{k}} u(t)\right\|_{W^{0}\left(\mathbf{R}^{m}\right)}^{2}\right) d t\right)^{1 / 2}
$$

For a proof, see [10], Theorem 7.4, Chapter 1. We identify the Sobolev space $W^{k}\left(\mathbf{R}^{m}\right)$ in the usual way with its Fourier transform, consisting of all functions $a(\xi)$ which are square integrable with respect to the weight $\left(1+|\xi|^{2}\right)^{k} d \xi$. Thus $u \in \mathscr{L}^{2}\left((0, \infty), W^{k}\left(\mathbf{R}^{m}\right)\right)$ is an $\mathscr{L}^{2}$ function with respect to $t$ with values in a weighted $\mathscr{L}^{2}$-space with respect to $\xi$, and the norm (5) becomes

$$
\|u\|_{\mathscr{L}^{2}\left((0, \infty), W^{k}\left(\mathbf{R}^{m}\right)\right)}=\left(\int_{0}^{\infty}\left(\int_{\mathbf{R}^{m}}|u(t, \xi)|^{2}\left(1+|\xi|^{2}\right)^{k} d \xi\right) d t\right)^{1 / 2} .
$$

After these preparations, we are ready to define the $R^{j}$. For $a \in$ $W^{-\infty}\left(\mathbf{R}^{m}\right)$, set

$$
R^{j} a(t, \xi):=\frac{t^{j}}{j !} \varphi\left(\beta_{j} t\left(1+|\xi|^{2}\right)^{1 / 2}\right) a(\xi)
$$

with

$$
\beta_{j}:=\max \left(1,\left(j^{2}\right) \alpha_{j}\right)
$$

$\varphi$ is a fixed $[0,1]$-valued function in $C_{0}^{\infty}(\mathbf{R})$, supported in $[-1,1]$, and identically 1 on $[-1 / 2,1 / 2]$.

Clearly, $R^{j} a \in W^{-\infty}(\Omega)$. Fix now $k \in \mathbf{N}$. Assume that $a \in$ $W^{k-j-1 / 2}\left(\mathbf{R}^{m}\right)$. To show (1), we shall use (4). For $t \in(0, \infty)$ fixed, the right-hand side of (8) is in $W^{k}\left(\mathbf{R}^{m}\right)$, since $a$ is locally integrable and $\varphi$ has compact support. So $R^{j} a$ is a $W^{k}\left(\mathbf{R}^{m}\right)$-valued function on $(0, \infty)$; it is easy to see that it is smooth. To estimate $\left\|R^{j} a\right\|_{W^{k}(\Omega)}$, we have to estimate the right-hand side of (6). The first contribution is (after squaring (6)):

$$
\begin{array}{r}
\int_{0}^{\infty}\left\|R^{j} a(t, \xi)\right\|_{W^{k}\left(\mathbf{R}^{m}\right)}^{2} d t=\int_{0}^{\infty}\left(\int_{\mathbf{R}^{m}}\left|R^{j} a(t, \xi)\right|^{2}\left(1+|\xi|^{2}\right)^{k} d \xi\right) d t \\
=\int_{0}^{\infty} \int_{\mathbf{R}^{m}} \frac{t^{2 j}}{(j !)^{2}} \varphi^{2}\left(\beta_{j} t\left(1+|\xi|^{2}\right)^{1 / 2}\right)|a(\xi)|^{2}\left(1+|\xi|^{2}\right)^{k} d \xi d t
\end{array}
$$


Since supp $\varphi \subset[-1,1]$, we are in fact only integrating over the subset of $(0, \infty) \times \mathbf{R}^{m}$ where

$$
\beta_{j} t\left(1+|\xi|^{2}\right)^{1 / 2} \leq 1
$$

Using this and Fubini's theorem, the last integral in (10) can be estimated by

$$
\begin{gathered}
\int_{\mathbf{R}^{m}}\left(\int_{0}^{\left(\beta_{j}\right)^{-1}\left(1+|\xi|^{2}\right)^{-1 / 2}} d t\right) \frac{\left(1+|\xi|^{2}\right)^{k}}{(j !)^{2}\left(\beta_{j}\right)^{2 j}\left(1+|\xi|^{2}\right)^{j}}|a(\xi)|^{2} d \xi \\
\quad=\frac{1}{\left(\beta_{j}\right)^{2 J+1}(j !)^{2}} \int_{\mathbf{R}^{m}}|a(\xi)|^{2}\left(1+|\xi|^{2}\right)^{k-j-1 / 2} d \xi \\
=\frac{1}{\left(\beta_{j}\right)^{2 j+1}(j !)^{2}}\|a\|_{W^{k-j-1 / 2}\left(\mathbf{R}^{m}\right) .}
\end{gathered}
$$

The calculation for the second contribution in (6) is essentially the same. Note that

$$
\begin{aligned}
\frac{\partial^{k}}{\partial t^{k}} R^{J} a= & \frac{\partial^{k}}{\partial t^{k}}\left(\frac{t^{j}}{j !} \varphi\left(\beta_{j} t\left(1+|\xi|^{2}\right)^{1 / 2}\right)\right) a(\xi) \\
= & \left(\sum_{s=0}^{k}\left(\begin{array}{c}
k \\
s
\end{array}\right) \frac{\partial^{s}}{\partial t^{s}}\left(\frac{t^{j}}{j !}\right)\left(\beta_{j}\left(1+|\xi|^{2}\right)^{1 / 2}\right)^{k-s}\right. \\
& \left.\times\left(\frac{\partial^{k-s}}{\partial t^{k-s}} \varphi\right)\left(\beta_{j} t\left(1+|\xi|^{2}\right)^{1 / 2}\right)\right) a(\xi) .
\end{aligned}
$$

Therefore, in order to estimate

$$
\int_{0}^{\infty} \int_{\mathbf{R}^{m}}\left|\left(\frac{\partial^{k}}{\partial t^{k}} R^{j} a\right)(t, \xi)\right|^{2} d \xi d t,
$$

it suffices to consider the terms

$$
\begin{aligned}
\int_{0}^{\infty} \int_{\mathbf{R}^{m}}\left(\frac{\partial^{s}}{\partial t^{s}}\left(\frac{t^{j}}{j !}\right)\right)^{2}\left(\left(\frac{\partial^{k-s}}{\partial t^{k-s}} \varphi\right)\left(\beta_{J} t\left(1+|\xi|^{2}\right)^{1 / 2}\right)\right)^{2} \\
\times \beta_{j}^{2 k-2 s}\left(1+|\xi|^{2}\right)^{k-s}|a(\xi)|^{2} d \xi d t
\end{aligned}
$$

For $s>j$, the integrand is 0 , so nothing needs to be estimated. For $s \leq j$, (14) may be treated exactly as (10), and we find that it is estimated by

$$
\frac{C_{k}}{\left(\beta_{j}\right)^{2 j-2 k+1}}\|a\|_{W^{k-j-1 / 2}\left(\mathbf{R}^{m}\right)}^{2},
$$


where $C_{k}$ is a constant depending on $k$ only. This shows property (i) of the theorem. From (12) and (15), we also read off that

$$
\left\|R^{J}\right\|_{k} \leq \frac{C_{k}}{\left(\beta_{j}\right)^{j-k}}
$$

with a different $C_{k}$ (note that $\left.\beta_{\jmath} \geq 1\right)$. Thus

$$
\sum_{j \geq 0}\left\|R^{j}\right\|_{k} \alpha_{j} \leq C_{k} \sum_{j \geq 0} \frac{\alpha_{j}}{\left(\beta_{j}\right)^{j-k}} .
$$

Now for $j>k,\left(\beta_{j}\right)^{j-k} \geq \beta_{j} \geq(j)^{2} \alpha_{j}$, so that the right-hand side of (17) is finite. This proves (iii).

To prove (ii), we first observe that the trace of $\partial^{s} R^{j} a / \partial t^{s}$ on $\mathbf{R}^{m}$ is the limit, in $W^{k-s-1 / 2}\left(\mathbf{R}^{m}\right)$, as $t_{0} \rightarrow 0^{+}$, of the traces on the hyperplanes $t=t_{0}([\mathbf{1 0}])$. Since $0 \leq s \leq k-1$, this convergence takes a fortiori place in $W^{0}\left(\mathbf{R}^{m}\right)=\mathscr{L}^{2}\left(\mathbf{R}^{m}\right)$. Thus the trace we look for is the $\mathscr{L}^{2}$-limit, as $t \rightarrow 0^{+}$, of

$$
\frac{\partial^{s}}{\partial t^{s}}\left(\frac{t^{j}}{j !} \varphi\left(\beta_{j} t\left(1+|\xi|^{2}\right)^{1 / 2}\right)\right) a(\xi) .
$$

Because the limit exists, we may calculate it by taking the pointwise limit a.e.; but this limit is $\delta_{s j} a(\xi)$, since for $t$ small enough, $\varphi\left(\beta_{j} t\left(1+|\xi|^{2}\right)^{1 / 2}\right)$ $\equiv 1$ (because $\xi$ is fixed). This proves (ii), and the proof of Theorem 3.1 is complete.

Acknowledgments. I am indebted to H. P. Boas for discussions concerning various aspects of the problem considered here. These led to substantial improvements over an earlier version of this paper. G. Komatsu has pointed out that the techniques from this earlier version could be used to obtain a constructive proof of Theorem 3.2 in [12]. Finally, I thank R. Meise for introducing me to the splitting theorem needed in Remark 2.1.

\section{REFERENCES}

[1] D. Barrett, Regularity of the Bergman projection on domains with transverse symmetries, Math. Ann., 258 (1982), 441-446.

[2] S. Bell, A duality theorem for harmonic functions, Michigan Math. J., 29 (1982), 123-128.

[3] A Sobolev inequality for pluriharmonic functions, Proc. Amer. Math. Soc., 85, Nr. 3 (1982), 350-352.

[4] , Boundary behavior of proper holomorphic mappings between non-pseudoconvex domains, Amer. J. Math., 106, Nr. 3 (1984), 639-643.

[5] S. Bell and H. Boas, Regularity of the Bergman projection in weakly pseudoconvex domains, Math. Ann., 257 (1981), 13-30. 
[6] S. Bell and D. Catlin, Boundary regularity of proper holomorphic mappings, Duke Math. J., 49 (1982), 385-396.

[7] K. Diederich and I. Lieb, Konvexität in der komplexen Analysis, DMV Seminar, Band 2, Birkhäuser 1981.

[8] L. Hörmander, Linear Partial Differential Operators, Grundl. d. math. Wissenschaften, Band 116, Springer 1976.

[9] G. Komatsu, Boundedness of the Bergman projector and Bell's duality theorem, Tôhoku Math. J., 36 (1984), 452-467.

[10] J. Lions and E. Magenes, Non-Homogeneous Boundary Value Problems and Applications I, Grundl. d. math. Wissenschaften, Band 181, Springer 1972.

[11] R. Seeley, Extension of $C^{\infty}$-functions defined in a half space, Proc. Amer. Math. Soc., 15, Nr. 4 (1964), 625-626.

[12] E. Straube, Harmonic and analytic functions admitting a distribution boundary value, Ann. Scuola Norm. Sup. Pisa, 11, Nr. 4 (1984), 559-591.

[13] D. Vogt, Subspaces and Quotient Spaces of ( $s$ ), in Functional Analysis; Surveys and Recent Results; Bierstedt K. and Fuchssteiner B. editors, North-Holland Mathematics Studies 27 (1977).

[14] Sequence Space Representations of Spaces of Test Functions and Distributions, in Functional Analysis, Holomorphy and Approximation Theory; Zapata G. editor, Lecture Notes in Pure and Appl. Math. 83, Marcel Dekker, 1983.

Received March 8, 1985 and in revised form June 10, 1985. Partially based on research supported by a grant from Swiss National Science Foundation. Also partially supported by an Indiana University Summer Faculty Fellowship

INDIANA UNIVERSITY

BLOOMINGTON, IN 47405 


\title{
PACIFIC JOURNAL OF MATHEMATICS EDITORS
}

\author{
V. S. VARADARAJAN \\ (Managing Editor) \\ University of California \\ Los Angeles, CA 90024 \\ HERBERT ClEMENS \\ University of Utah \\ Salt Lake City, UT 84112 \\ R. FINN \\ Stanford University \\ Stanford, CA 94305
}

\author{
HERMANN FLASCHKA \\ University of Arizona \\ Tucson, AZ 85721 \\ RAMESH A. GANGOLLI \\ University of Washington \\ Seattle, WA 98195 \\ VAUGHAN F. R. JONES \\ University of California \\ Berkeley, CA 94720 \\ ROBION KIRBY \\ University of California \\ Berkeley, CA 94720
}

C. C. MOORE

University of California

Berkeley, CA 94720

H. SAMELSON

Stanford University

Stanford, CA 94305

HAROLD STARK

University of California, San Diego

La Jolla, CA 92093

\section{ASSOCIATE EDITORS}

\author{
R. AREnS \\ E. F. BECKENBACH \\ B. H. NEUMANN \\ F. WOLF \\ K. YOSHIDA \\ (1906-1982)

\section{SUPPORTING INSTITUTIONS}

\begin{abstract}
UNIVERSITY OF ARIZONA
UNIVERSITY OF BRITISH COLUMBIA

UNIVERSITY OF CALIFORNIA

MONTANA STATE UNIVERSITY

UNIVERSITY OF NEVADA, RENO

NEW MEXICO STATE UNIVERSITY

OREGON STATE UNIVERSITY
\end{abstract} \\ CALIFORNIA INSTITUTE OF TECHNOLOGY \\ UNIVERSITY OF OREGON \\ UNIVERSITY OF SOUTHERN CALIFORNIA \\ STANFORD UNIVERSITY \\ UNIVERSITY OF HAWAII \\ UNIVERSITY OF TOKYO \\ UNIVERSITY OF UTAH \\ WASHINGTON STATE UNIVERSITY \\ UNIVERSITY OF WASHINGTON
}

The Supporting Institutions listed above contribute to the cost of publication of this Journal, but they are not owners or publishers and have no responsibility for its content or policies.

Mathematical papers intended for publication in the Pacific Journal of Mathematics should be in typed form or offset-reproduced (not dittoed), double spaced with large margins. Please do not use built up fractions in the text of the manuscript. However, you may use them in the displayed equations. Underline Greek letters in red, German in green, and script in blue. The first paragraph must be capable of being used separately as a synopsis of the entire paper. In particular it should contain no bibliographic references. Please propose a heading for the odd numbered pages of less than 35 characters. Manuscripts, in triplicate, may be sent to any one of the editors. Please classify according to the scheme of Math. Reviews, Index to Vol. 39. Supply name and address of author to whom proofs should be sent. All other communications should be addressed to the managing editor, or Elaine Barth, University of California, Los Angeles, California 90024.

There are page-charges associated with articles appearing in the Pacific Journal of Mathematics. These charges are expected to be paid by the author's University, Government Agency or Company. If the author or authors do not have access to such Institutional support these charges are waived. Single authors will receive 50 free reprints; joint authors will receive a total of 100 free reprints. Additional copies may be obtained at cost in multiples of 50 .

The Pacific Journal of Mathematics is issued monthly as of January 1966. Regular subscription rate: $\$ 190.00$ a year (5 Vols., 10 issues). Special rate: $\$ 95.00$ a year to individual members of supporting institutions.

Subscriptions, orders for numbers issued in the last three calendar years, and changes of address should be sent to Pacific Journal of Mathematics, P.O. Box 969, Carmel Valley, CA 93924, U.S.A. Old back numbers obtainable from Kraus Periodicals Co., Route 100, Millwood, NY 10546.

The Pacific Journal of Mathematics at P.O. Box 969, Carmel Valley, CA 93924 (ISSN 0030-8730) publishes 5 volumes per year. Application to mail at Second-class postage rates is pending at Carmel Valley, California, and additional mailing offices. Postmaster: send address changes to Pacific Journal of Mathematics, P.O. Box 969, Carmel Valley, CA 93924.

PUBLISHED BY PACIFIC JOURNAL OF MATHEMATICS, A NON-PROFIT CORPORATION

Copyright (C) 1986 by Pacific Journal of Mathematics 


\section{Pacific Journal of Mathematics}

\section{Vol. 123, No. $2 \quad$ April, 1986}

David Jay Anick, A loop space whose homology has torsion of all orders . . 257 Steven P. Diaz, Space curves that intersect often ................. 263

Thierry Fack and Hideki Kosaki, Generalized $s$-numbers of $\tau$-measurable

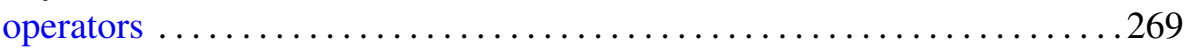

Karl Heinrich Hofmann and Karl Strambach, Lie's fundamental

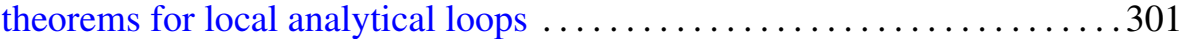

James Secord Howland, On the Kato-Rosenblum theorem ............. 329

Frieder Knüppel and Edzard Salow, Plane elliptic geometry over rings . . . . 337

Alan Noell, Peak points in boundaries not of finite type ................ 385

William J. Ralph, An extension of singular homology to Banach

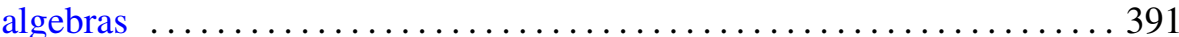

Wade C. Ramey, Averaging properties of pluriharmonic boundary values . . 407

Thomas Joseph Ransford, On the range of an analytic multivalued

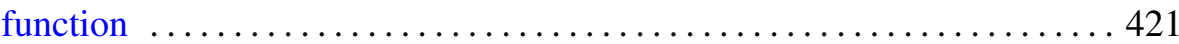

Christopher Donald Sogge, On restriction theorems of maximal-type . . . . 441

Edwin Spanier, Cohomology with supports $\ldots \ldots \ldots \ldots \ldots \ldots \ldots \ldots \ldots 47$

Emil J. Straube, Orthogonal projections onto subspaces of the harmonic

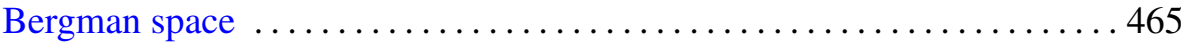

Thomas Vogel, Asymptotic behavior of two semilinear elliptic free boundary problems $\ldots \ldots \ldots \ldots \ldots \ldots \ldots \ldots \ldots$ 